\title{
Comunicado de la Universidad de Nariño
}

\section{A LA COMUNIDAD CIENTÍFICA COLOMBIANA}

En estos momentos cruciales en los que la promoción de la investigación en ciencia y en tecnología es un requerimiento indispensable para que las regiones y el país comiencen a superar los estadios precarios de desarrollo social y económico y las extremas desventajas geopolíticas, preocupa profundamente el futuro de la financiación de los proyectos que le apuntan a la solución de necesidades prioritarias, a través de la generación y la aplicación de conocimiento y de la innovación productiva y social. La disminución ostensible del presupuesto de COLCIENCIAS para el año 2018, no es consecuente con el compromiso demostrado por los investigadores de fortalecer la apropiación del conocimiento entre los diferentes grupos sociales y las comunidades académicas, compromiso que ha generado logros producto de un trabajo arduo y condiciones rigurosas de calidad.

Colombia ha sido un país que no dispone de un número importante de fuentes de financiación para la ciencia y la tecnología; los recursos que el gobierno nacional le asigna a COLCIENCIAS, a los escasos Institutos de investigación y a las Universidades son exiguos e irrelevantes para fortalecer e incentivar la investigación en el contexto nacional. Esta situación impide que la investigación alcance los niveles que todos esperamos y conlleva a un campo restringido de visibilidad científica, la cual sólo se logra con el esfuerzo personal e institucional de quienes tienen la posibilidad de acceder a los recursos; las iniciativas planteadas por quienes retornan a nuestro territorio, con formación académica de alto nivel se truncan y el sueño de tener a "Colombia la más educada", es simplemente un eslogan coyuntural.

La inversión para la innovación, la ciencia, la tecnología y, en general, para fortalecer los procesos de investigación y formación de investigadores, cada día se ve más reducida, esta circunstancia propicia la salida de jóvenes talentosos e investigadores reconocidos, quienes se ven obligados a desarrollar las actividades profesional y postgraduada en otros lugares del mundo, incrementando el desarraigo y reduciendo la posibilidad de aprovechar la formación académica y la capacidad de trabajo en favor de la sociedad colombiana.

El recorte presupuestal planteado por el gobierno para COLCIENCIAS, para el año2018 pone en riesgo la continuidad de los proyectos de Ciencia y Tecnología que le han aportado al país, minimiza la posibilidad de proponer nuevas estrategias de innovación y sitúa en desventaja a las regiones e instituciones que no disponen de recursos para financiar las iniciativas de investigación enfocadas a la solución de problemas que afectan, en buena medida, a las comunidades más vulnerables. Además, la imposibilidad de financiar la investigación básica influye directamente en el mínimo desarrollo de la investigación aplicada y, por ende, induce a un bajo impacto de la investigación en la superación de factores de atraso de la sociedad.

Considerando a la Educación como el camino para lograr la paz y la equidad nacional, los investigadores, los grupos de investigación, los centros e institutos de investigación adscritos 
a instituciones de Educación Superior solicitamos al Gobierno Nacional y, específicamente, a los responsables de la asignación de recursos al Presupuesto de COLCIENCIAS reconsiderar la política de desfinanciación de esta Entidad y, en cambio, garantizar las condiciones adecuadas que propicien el pleno funcionamiento y que permitan contribuir al desarrollo del país con el aporte de la investigación en Ciencia, Tecnología e Innovación, en beneficio de todos los colombianos.

CONSEJO ACADÉMICO Y DIRECTIVAS ACADÉMICAS UNIVERSIDAD DE NARIÑO 